\title{
10. Smartphone challenges to stimulate cycling: clues from a living lab in Enschede
}

\author{
Tom Thomas, Bingyuan Huang, Benjamin \\ Groenewolt and Eric C. van Berkum
}

\subsection{INTRODUCTION}

During the past century, population and wealth growth have led to the creation of large urban areas in which people and goods are mainly connected by road transport. This has led to urban problems such as congestion and pollution. Public transport can reduce road transport, but the question is whether mass transit can transform cities to a human scale. Together with walking, cycling may be indispensable to develop liveable human scale cities. Through cycling, people can in principle access all locations in neighbourhoods, and thus will be able to connect with their urban environment physically. It is a local activity in itself, it is healthy, and it is more than simply travelling from A to B. It is therefore important to stimulate cycling.

Positive interventions or 'soft measures', such as personal travel planning, subsidies, providing feedback, rewards, public transport (PT) discount could stimulate the use of sustainable transport options (e.g., Bamberg and Schmidt, 2003; Cairns et al., 2008). In the age of big data, mobile phones and software platforms are becoming useful tools to collect travel behaviour data and deliver interventions. Global Positioning System (GPS) data from smartphones can be used to estimate travel modes and travel routes accurately (e.g., Biljecki et al., 2013). Moreover, several research projects have used smartphones to deliver interventions (e.g., Bie et al., 2012; Poslad et al., 2015). These interventions include personalized feedback, self-monitoring, challenges and goal-setting, social comparison and rewards.

Regarding cycling, Weber et al. (2018), for example, have shown that cycling campaigns in the United States, United Kingdom (UK) and Australia can attract many (potential) cyclists using gamification in smartphone apps. There are also several cycling apps that have a seamless server to end-users, 
attracting thousands of users over a longer period of time (sometimes years). For example, Strava (www.strava.com) works as a fitness app that tracks and analyses cycling or running trips, explores new routes and provides the social network to compete with friends. Apps such as BetterPoints (www .betterpoints.uk/page/qr) and SMART (www.smartintwente.nl) explicitly reward users when they travel (more) sustainably. Although the latter apps are not only focused on cycling, they also attract occasional cyclists who only cycle now and then. Their aim, therefore, is to encourage behaviour change among all travellers.

Unfortunately, there are few scientific studies about the effects of rewards on cycling. Most studies in this field focus on avoiding rush-hour travelling (e.g., Kumar et al., 2016; Yang and Tang, 2018), and some studies have looked into the effect of rewards on PT ridership (e.g., Abou-Zeid and Fujii, 2016). There are a few exceptions. In separate studies in Palermo (Di Dio et al., 2018) and Vienna and Birmingham (Tsirimpa et al., 2019), apps were used to reward sustainable transport, including cycling. The amount of cycling almost doubled or even more than doubled, respectively, as a result. Although both studies have a clear experimental design, including a baseline measurement, the samples are relatively small (77 commuters and 64 individuals, respectively). The drawback of these experiments is that participants know they are participating, which could affect their behaviour. Moreover, self-selection could be an issue, because participants are more likely to have a positive attitude towards the aim of the experiment, that is, encouraging cycling.

In this study, we take another approach. We observe the behaviour of all users of the SMART app in the Dutch region of Twente. In this chapter, we focus on multiple and targeted challenges that are provided monthly. The advantage is that users are not influenced by an experimental setting and that the sample is much larger (an order of magnitude, that is, about a factor of ten) than in the studies mentioned above. Moreover, the measurements are done over a relatively long period. Some of the challenges have been repeated, and as a result, we are better able to establish which type of challenge is effective. As this study is not a real experiment, there are some drawbacks that will be discussed as well. The most important results from observation between March 2017 and February 2018 are summarized in this chapter.

\subsection{METHOD}

SMART tracks travel behaviour change of users, similar to BetterPoints. Modes are deduced using probabilistic Bayesian mode deduction models, which were trained on a complete training set (consisting of all types of users and types of trips) of about 3000 trips. The success rate of mode detection is 82 per cent (Thomas et al., 2018), which is comparable to similar applications 
(e.g., Rasmussen et al., 2015). Note that wrong detections are hardly an issue among SMART users, and will not influence the results, because the detection errors are not systematic (such as too few or too many detected bike trips).

The positive incentives were provided through the SMART app. The app is not only rewarding sustainable travel but also has a wider scope, which is illustrated by the four main functionalities in Figure 10.1. The left panel of Figure 10.1 depicts the SMART app dashboard. From top to bottom, the figure depicts feedback on historical travel patterns, challenges, rewards, and social/ group incentives. Users can explore all functions of the app from this page. The figures in the right panel are screenshots from other pages of the SMART app. The first functionality is travelling information. This can be actual traffic information in which users are notified in case of road works or large-scale events, but the app also provides the actual historic travel pattern of the user (upper panel). This information makes users more aware of their current behaviour, which could be a trigger for behaviour change. The second functionality (second panel) are challenges which users commit themselves, that is, they need to fulfil a challenge during the challenge period. The commitment to fulfil a challenge may be enhanced when users get rewarded upon completion of the challenge. When the challenge is fulfilled, the system will immediately give the corresponding amount of points. The earned points can then be redeemed for various discounted products and services (third panel). Incentive providers include local shops such as cafeterias, restaurants and bike shops, but also leisure attractions such as the local ice rink. Finally, social incentives to encourage cycling (bottom panel) are also in the SMART app. This includes a competition with rankings to compare behaviour with others, and group challenges in which participants can invite friends to fulfil challenges together.

In this chapter, we explore the effect of monthly bike challenges. We included three main types of choice challenges with a challenging period of 14 days. These are location challenges, that is, number of cycling trips to a fixed location; cumulative challenges, that is, number of total cycling trips or cycling kilometres; and rate challenges, that is, number of days to ride at least 10 kilometres by bike or number of kilometres to be ridden by bike on at least ten out of the 14 days. The different types of challenges were designed and distributed throughout the year to see which type is more effective.

There are different ways to design challenges. One approach is to reward users when they cycle more compared to the baseline. This stimulates real change, as participants have to improve to be rewarded, but this may also discourage participation (Matushkina and Nevalennaya, 2010). Moreover, it only rewards behavioural change, which is unfair to travellers who already cycle a lot. Another approach is to reward users for each mile they cycle (for example, BetterPoints). The burden for the user is relatively low. However, some studies showed that targeted and challenging goals led to higher per- 

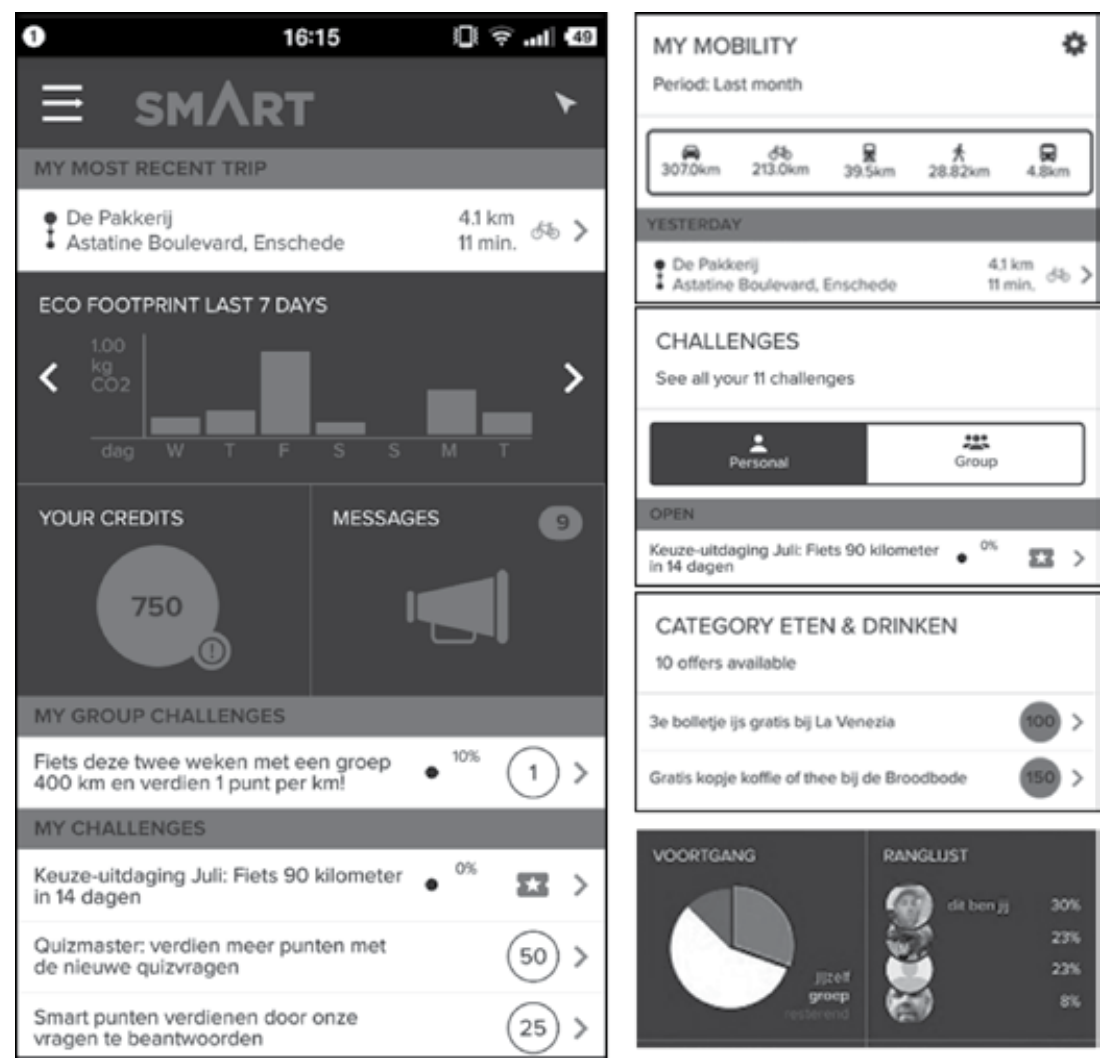

Figure 10.1 SMART dashboard (left) and functionalities (right)

formance than easy or 'do your best' goals (Locke and Latham, 2002). In our design, we tried to combine the advantages of both approaches by introducing choice challenges. Users themselves have the option to choose one out of five levels, from very easy to very difficult. This enables us to provide equal opportunities for everyone and at the same time personalize the challenge level in which participants can challenge themselves. As participants can choose their own level, it is also less likely that they will be discouraged to participate because a challenge is too difficult.

One of the drawbacks of choice challenges is that users can still choose a challenge and win points without changing their behaviour. In that case they choose a level that is too easy for them. To stimulate the choice of difficult challenges, participants get higher rewards when they accomplish more difficult challenges. The reward is proportional to the required number of bike trips 
(12 SMART points per trip longer than $2 \mathrm{~km}$ ) or total required bike kilometres (4 SMART points per $\mathrm{km}$ ). However, for each challenge 30 SMART points could also be earned to motivate car users to join the (easiest) challenge. No reward is given if the participant cannot complete the chosen challenge. Therefore, participants have to choose between an easy challenge with relatively fewer points but a greater chance to be rewarded, or a difficult challenge with relatively more points but a higher chance of failing.

In the results, we compare trips within and outside the challenge period. It is important to emphasize that every month one monthly choice challenge was offered, but that the duration of the challenge was only 14 days. Therefore, a comparison during the challenge and outside the challenge period is possible for each month. We evaluated the difference between inside and outside challenge periods in terms of distance-based mode share. This is the percentage of the total distance that is covered by each mode during each measurement period. We used distance rather than trip frequency, because the latter might be disproportionately influenced by people shifting from walking to cycling for the shortest trips. To avoid the disproportionally large contribution of long car trips in the distance-based mode shares, we only included trips below 20 $\mathrm{km}$. For larger distances, cycling is not considered as a viable option (Fioreze et al., 2018).

Based on individual mode share differences (between inside and outside challenge period), we estimated the standard error in the average mode share difference (weighted by the total covered distance per participant) as the standard deviation of the mean. In the results, we illustrate the uncertainties by two times the standard error.

\subsection{USERS}

Most SMART users are inhabitants of the city of Enschede, which is a medium-sized Dutch city with about 160000 inhabitants. The modal split (based on trip frequency) of Enschede is quite representative for the Netherlands (55 per cent car, 26 per cent bike, 15 per cent walk, and 4 per cent PT according to EPOMM 2020). We recruited users via specific campaigns, such as the Enschede Cycle City campaigns, Charity campaigns (to cycle for a charity), Bike2Sport campaigns that stimulate teams to use the bike to sports events, and the SMART green campaign in which SMART cyclists get a faster change to a green light when they approach a certain set of traffic lights. However, there is also a general inflow due to various media and recommendations by friends.

Once new users have installed SMART, they could immediately use all functionalities and participate in challenges. They also did not need to provide extra information (such as age or gender). In other words, they were not 
recruited for an experiment, but simply used the app because they wanted to. Thus, we created a realistic real-life context to analyse travel behaviour. However, as a drawback, we have no demographic information of the users, and we were not able to do a 'before' measurement to establish the baseline behaviour.

In Figure 10.2, we show the number of active users per day and month. Active users are defined as users who have at least one recorded trip per day (number of daily users) or during ten days in a month (number of monthly users). Note that some people may not make a trip at all during a given day (zero trips), and therefore do not record a trip even when they use SMART. The darker gray upper line shows the number of daily users when we correct for this effect. The figure shows some interesting results. The many dips in the number of active daily users correspond with the weekends in which the rate of zero trips is larger than during workdays. Except for these dips, the number of daily and monthly users is quite stable and increases rapidly at the start of 2018. This rapid ascent can be attributed to the success of the Cycling City campaigns. One of the attractive parts of the campaign is that users get automatically one point per cycling or walking kilometre without the need to participate in cycling or walking challenges.

About 50 per cent of the users participated at least once in a monthly challenge. Although the turnover is quite low, some users dropped out during this period. If we also include those users, in the end about 1000 users participated in a challenge. This is quite a substantive sample (compared to other studies). However, users do not always participate. They sometimes skip monthly challenges or stop participating in them. On a monthly basis, about 30 per cent of the users participated on average. There is also a difference between participants and non-participants, indicating a self-selection effect. Car share (distance-based for trips shorter than $20 \mathrm{~km}$ ) is 52 per cent among users who did not participate in monthly challenges. This is quite comparable with the car share of the population. However, outside the challenge period, the car share drops to 35 per cent among users who participate in challenges. Even so, the participants still have a significant car share, that has the potential to get further decreased. Note that it is important to realize that there are few pure car users in the Netherlands: almost all Dutch people are occasional cyclists, that is, the bike share of an individual is seldom zero or 100 per cent. Therefore, the bike share could still be further increased for most of the participants.

\subsection{EFFECTS OF DIFFERENT CHALLENGES}

In the upper panel of Figure 10.3, we show the difference in modal shares between the challenge period and the outside challenge period. The figure clearly shows a significant increase in the bike share and a reduction in the car 


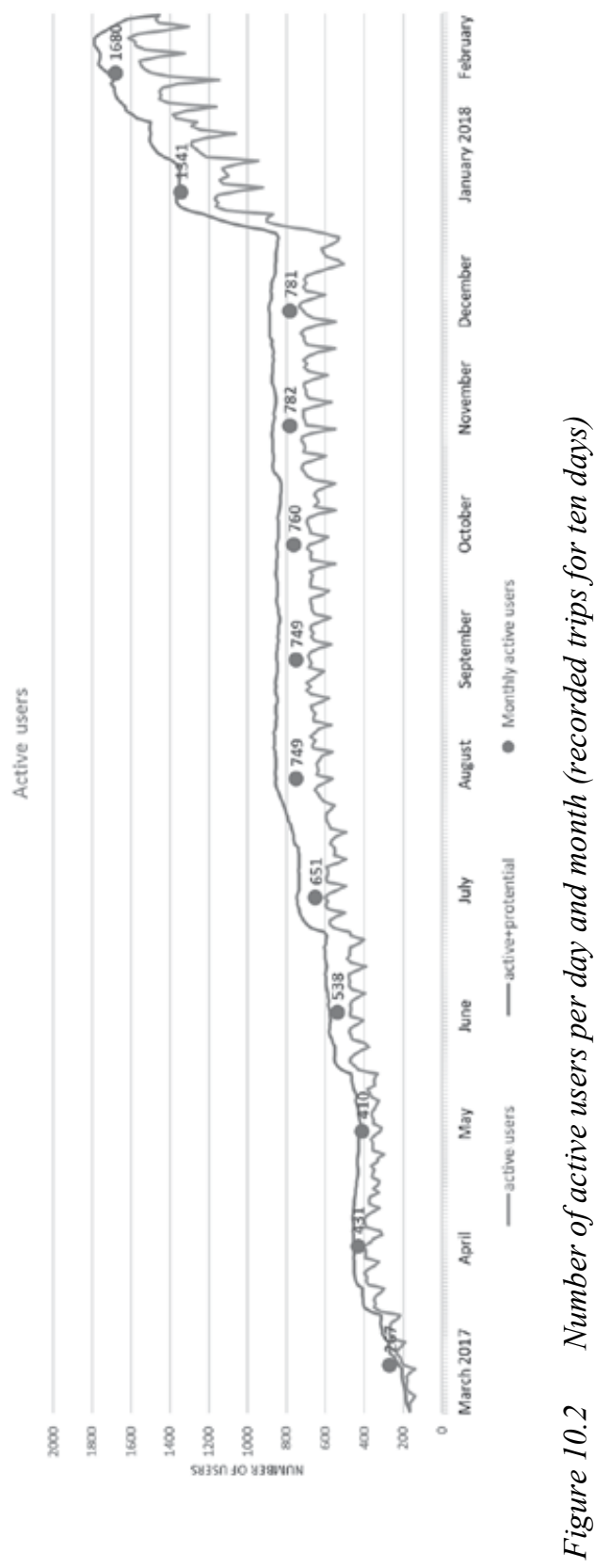


share during the challenge period, except for the location challenges. Note that location challenges could be perceived as untargeted when participants have to visit a location they have never visited before. Because we consider many months, we can safely attribute observed changes to the challenges and not to other external temporal effects such as weather. We checked this by using the non-participants as a control group. Unsurprisingly, they did not show any significant change in modal shares between weeks when most participants were in the challenge period and the weeks outside the challenge periods.

The figure shows that challenges with high completion rates are not less effective. On the contrary, although not statistically significant, Figure 10.3 indicates that challenges with the highest completion rates also yield most of the behavioural change. The error bars illustrate two times the standard error in the difference of the mode shares. Interestingly, easy and difficult challenges (relative to the baseline) yield more or less the same behaviour change. For the completed challenges, the increase in cycling is almost twice as high for difficult challenges. However, when challenges are not completed there is almost no behaviour change. While almost all the easy challenges are completed, almost half of the difficult challenges are not completed. The fact that behavioural change is relatively significant when participants complete a difficult challenge is compensated by the fact that difficult challenges are completed much less frequently.

This result was not expected in advance. Participants who choose easy challenges do not need to change their behaviour to complete the challenge. The fact that they did suggests that the challenges in themselves encourage participants to change their behaviour. Perhaps participants are more aware of their behaviour when they participate in a challenge, and therefore are more likely to change their behaviour even when they can complete the challenge without behavioural change. This result is promising for challenges in which users can choose the difficulty level themselves.

Compared with Di Dio et al. (2018) and Tsirimpa et al. (2019), the behavioural change appears to be quite moderate. In Palermo, the cycling share increased from 5 per cent to 9 per cent (Di Dio et al., 2018). While the changes in percentage points are comparable, they start from a much lower base. Relative changes in bike shares are much larger in those two studies. Of course, it is harder to achieve large relative changes from a higher base. Therefore, it is hard to compare results from the Netherlands with those in the UK or Italy. However, their observed effects still appear to be quite strong compared to ours. As mentioned earlier, it is possible that self-selection is more of an issue in those types of studies, which results in overestimating the potential effects. 


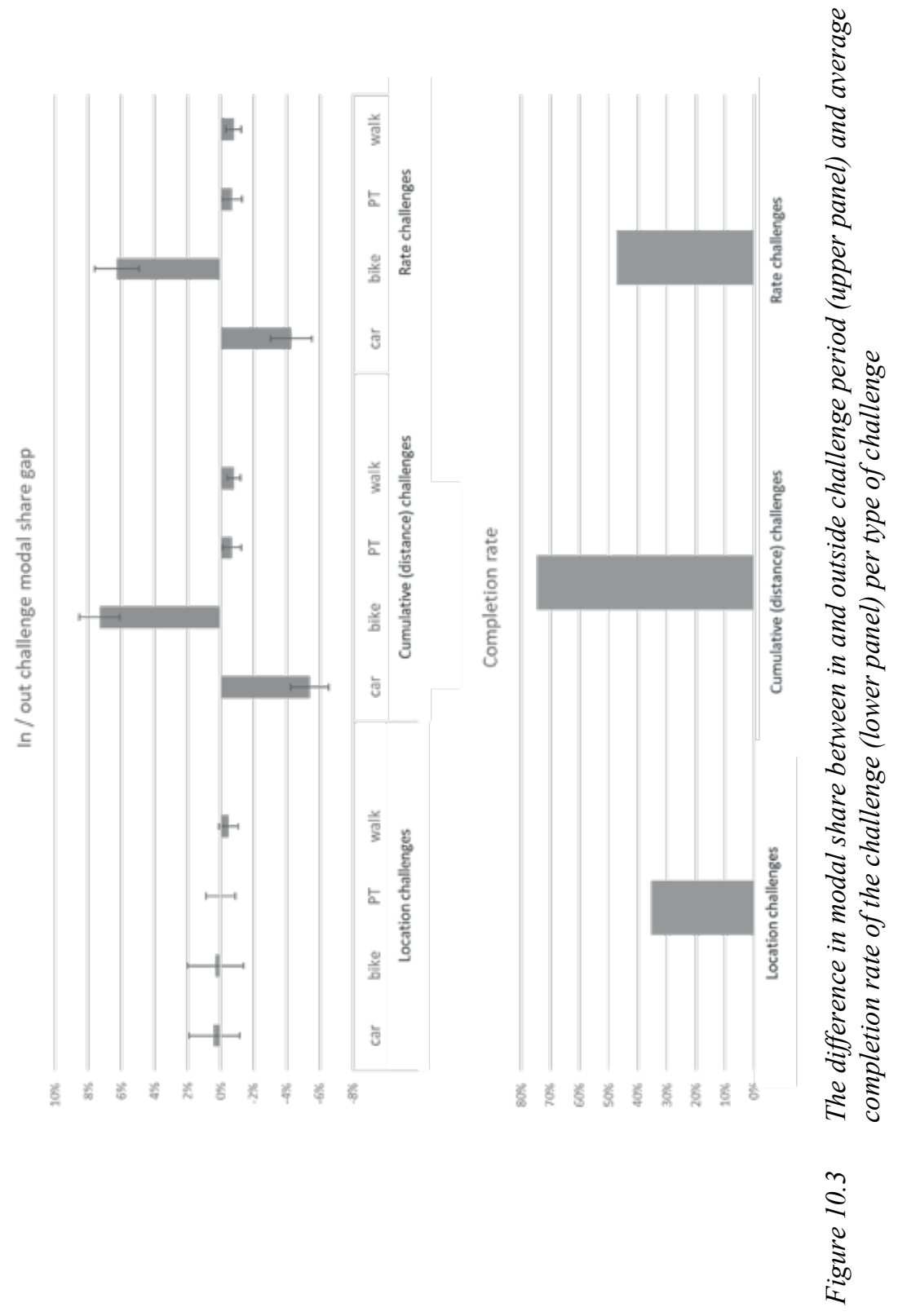




\subsection{CONCLUSION}

We conclude that monthly choice challenges can be an effective way to encourage cycling, although effects may be more moderate than expected based on other small-scale experiments. Challenges that are easy to accomplish do not necessarily yield less behavioural change. On the contrary, our result suggests that it is not necessary to request users to improve their behaviour compared to a (historic) baseline. However, these results are still on a highly aggregated level. In the next step, we will use an individual data and modelling approach to improve our analysis. One of the important remaining questions is whether the behavioural change will be sustained when no challenges are provided. Or do people fall back towards their former behaviour, even if they say they will continue cycling more? In other words, do people cycle more over time, also outside the challenge periods? And how long should we provide users with these types of challenges to obtain sustained behaviour change? To answer this question, we not only need to look at individual data, but probably also need to extend the total observation period beyond one year.

\section{ACKNOWLEDGEMENTS}

This research is funded within EMPOWER as part of a European Union HORIZON 2020 programme.

\section{REFERENCES}

Abou-Zeid, M., and Fujii, S. (2016). Travel satisfaction effects of changes in public transport usage. Transportation, 43(2), 301-314.

Bamberg, S., and Schmidt, P. (2003). Incentives, morality, or habit? Predicting students' car use for university routes with the models of Ajzen, Schwartz, and Triandis. Environment and Behavior, 35(2), 264-285.

Bie, J., Bijlsma, M., Broll, G., Cao, H., Hjalmarsson, A., et al. (2012). Move better with tripzoom. International Journal on Advances in Life Sciences, 4(3), 125-135.

Biljecki, F., Ledoux, H., and Van Oosterom, P. (2013). Transportation mode-based segmentation and classification of movement trajectories. International Journal of Geographical Information Science, 27(2), 385-407.

Cairns, S., Sloman, L., Newson, C., Anable, J., Kirkbridge, A., and Goodwin, P. (2008). Smarter choices: assessing the potential to achieve traffic reductions using 'soft measures'. Transp. Reviews, 28(5), 593-618.

Di Dio, S., La Gennusa, M., Peri, G., Rizzo, G., and Vinci, I. (2018). Involving people in the building up of smart and sustainable cities: how to influence commuters' behaviors through a mobile app game. Sustainable Cities and Society, 42, 325-336.

EPOMM (2020). TEMS The EPOMM Modal Split Tool. Retrieved 11 June 2020 from http://tems.epomm.eu/. 
Fioreze, T., Thomas, T., Huang, B., and van Berkum, E. (2018). How employees view smart cycling to work: a regional survey in the Netherlands. Travel Behaviour and Society, 16, 224-234.

Kumar, V., Bhat, C.R., Pendyala, R.M., You, D., Ben-Elia, E., and Ettema, D. (2016). Impacts of incentive-based intervention on peak period traffic experience from the Netherlands. Transportation Research Record: Journal of the Transportation Research Board, 2543, 166-175.

Locke, E.A., and Latham, G.P. (2002). Building a practically useful theory of goal setting and task motivation: a 35-year odyssey. American Psychologist, 57(9), 705-717.

Matushkina, E., and Nevalennaya, A. (2010). Motivating contributions to Commute Greener! Nature of motivation and motivation loss. Master's thesis. Retrieved from https://gupea.ub.gu.se/bitstream/2077/24709/1/gupea_2077_24709_1.pdf.

Poslad, S., Ma, A., Wang, Z., and Mei, H. (2015). Using a smart city IOTT to incentivise and target shifts in mobility behaviour: is it a piece of pie? Sensors (Switzerland), 15(6), 13069-13096.

Rasmussen, T., Ingvardson, J., Halldórsdóttir, K., and Nielsen, O. (2015). Improved methods to deduct trip legs and mode from travel surveys using wearable GPS devices: a case study from the greater Copenhagen area. Computers, Environment and Urban Systems, 54, 301-313.

Thomas, T., Geurs, K.T., Koolwaaij, J., and Bijlsma, M. (2018). Automatic trip detection with the Dutch Mobile Mobility Panel: towards reliable multiple-week trip registration for large samples. Journal of Urban Technology, 25(2), 143-161.

Tsirimpa, A., Polydoropoulou, A., Pagoni, I. and Tsouros. I. (2019). A reward-based instrument for promoting multimodality. Transportation Research Part F, 65, 121-140.

Weber, J., Azad, M., Riggs, W., and Cherry, C.R. (2018). The convergence of smartphone apps, gamification and competition to increase cycling. Transportation Research Part F, 56, 333-343.

Yang, H., and Tang, Y. (2018). Managing rail transit peak-hour congestion with a fare-reward scheme. Transportation Research Part B: Methodological, 110, $122-136$. 